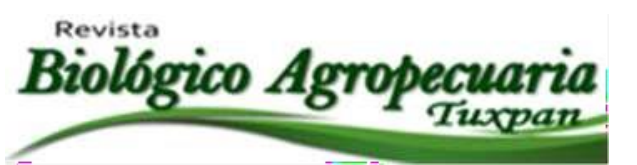

ISSN 2007-6940

Editorial Agricola Tux pan

https:/doi.org/10.47808/revistabioagro.v6i2.205
Vol. 6 No. 2

Julio-Diciembre

2018

\title{
Validación del método de elaboración tradicional del extracto de vainilla (Vanilla planifolia A.) en la Sierra de Otontepec
}

Validation of the traditional elaboration method of vanilla extract (Vanilla planifolia A.) in the Sierra de Otontepec

Solares, del A. C. D. ${ }^{1}$ Elorza, Martínez. P. ${ }^{1 凶}$; Rodríguez, B. A. ${ }^{1}$; López, C. R. D ${ }^{1}$; Aguirre, López. E. ${ }^{1}$

${ }^{1}$ Facultad de ciencias Biológicas y Agropecuarias, Universidad Veracruzana. Campus Tuxpan.

${ }^{\square}$ Autor para correspondencia: pelorza@uv.mx .

Recibido: 25/09/2018

Aceptado: 18/12/2018

\section{RESUMEN}

El objetivo del trabajo fue evaluar el método de elaboración tradicional de los extractos elaborados con refino artesanal, procedentes de la región de la Sierra de Otontepec Veracruz, con el propósito de conocer sus características y generar información para determinar las ventajas de la concentración de vainillina que presenta cada una de las muestras analizadas. Se obtuvieron los perfiles químicos y se identificaron compuestos químicos utilizando columnas cromatografías además del perfil químico general, encontrando que el contenido de alcohol en los extractos, que elaboran los productores cuenta únicamente con el 14\% de alcohol y lo mencionado en la NMX-FF-074-1996 dice que no debe ser menor del 30\% por lo que no cumple con este requisito. Con respecto al contenido de vainillina los contenidos variaron de 348 a un 8,447 \% (pm) por lo que cumplen con la Norma Oficial Mexicana que señala que el contenido mínimo de los extractos debe ser de 2,49\% en adelante, por lo tanto los extractos regionales se encuentran dentro de los parámetros y son aptos para su venta.

Palabras clave: cromatografía, extracto, Vanilla, Vainillina, Espectrofotometría

\section{ABSTRACT:}

The objective of the work was to evaluate the method of traditional elaboration of the extracts elaborated with artisanal refinement, coming from the region of the Sierra de Otontepec Veracruz, in order to know its characteristics and generate information to determine the advantages of the concentration of vanillin that presents each of the samples analyzed. The chemical profiles were obtained and chemical compounds were identified using chromatography columns in addition to the general chemical profile,

\section{Revista Científica Biológico Agropecuaria Tuxpan 6 (2)}

ISSN: 2007-6940 
finding that the alcohol content in the extracts, which are produced by the producers, only has $14 \%$ alcohol and the aforementioned in the NMX-FF- 074-1996 says that it should not be less than $30 \%$ so it does not meet this requirement. Regarding the content of vanillin, the contents ranged from 348 to $8.447 \%$ (pm), which is why they comply with the Official Mexican Standard that states that the minimum content of the extracts must be $2.49 \%$ upwards, therefore the Regional extracts are within the parameters and are suitable for sale.

Keywords: chromatography, extract, Vanilla, Vanillin, Spectrophotometry.

\section{INTRODUCCIÓN}

La Vainilla se ha cultivado desde tiempos prehispánicos en la región Totonaca del norte de Veracruz y Puebla. Los mayas y aztecas la utilizaban para enriquecer una bebida de cacao destinada a nobles y guerreros. La mayor producción se concentraba entre los totonacos alrededor de Veracruz y Papantla. Tiene su centro de origen en México (Soto, 2006). Aunque no se tiene conocimiento de su distribución original, pero es una orquídea de clima cálido húmedo que se ha registrado en la Sierra Madre de Oaxaca, en la Selva Lacandona en Chiapas y en el sur de Quintana Roo (Soto, 2009). Recibió el nombre de "vainilla" por los españoles, debido a que sus frutos, de entre $15 \mathrm{y}$ $30 \mathrm{~cm}$ de largo, se parecen a las vainas de espadas. Sus flores son visitadas por las pequeñas abejas de las orquídeas (Euglossini) entre febrero y mayo. Se considera que las semillas de frutos fragantes son dispersadas por murciélagos. Es un bejuco epífito de flores amarillas verdosas. También se conoce con los nombres náhuatl ixtlilxochitl o tlilxóchitl (flor negra), totonaco xanat o shanat, caxixanath y su chel (Soto, 2006).

Los extractos y esencias que se fabrican de la vainilla, son unos de los más importantes saborizantes naturales utilizados en la industria de las bebidas y de las confituras, así como domésticamente. Las vainas que se comercializan consisten en los frutos inmaduros, completamente desarrollados, y curados, de la planta denominada Vainilla planifolia, una orquídea colgante originaria de los bosques lluviosos de América Central, particularmente de México; de donde aún provienen las vainas de mejor calidad. El procesamiento del curado da inicio con las vainas recién cosechadas (CNP, 2000). La finalidad del curado es detener el proceso vegetativo natural y acelerar los cambios que llevarán a la formación de los constituyentes aromáticos del sabor. El método de la elaboración del curado difiere entre las diversas áreas de producción y esto puede asumir una influencia muy importante en la variación de la calidad y del perfil aromático de las vainas que se comercializan. Los usos más importantes de la vainilla son los siguientes (Curti, 1995) 1. Industria alimenticia como saborizante y aromatizante, en la elaboración de helados, chocolotes, dulces, pasteles, atoles y repostería en general. 2. Industria licorera y refresquera. 3. Industria de cosméticos y perfumería. 4. Industria tabacalera, como aromatizante de cigarros y puros. 5. Artesanías. El beneficiado de la vainilla es un proceso de fermentación, mediante el cual los frutos verdes,

Revista Científica Biológico Agropecuaria Tuxpan 6 (2) 
los cuales carecen de aroma, se modifican drásticamente hasta exhibir un color café oscuro $\mathrm{y}$ brillante y perfiles de aroma y sabor, provenientes de una mezcla de cientos de compuestos donde destaca la vainillina (ReyesLópez et al., 2008). Estos cambios se atribuyen a una serie de reacciones químicas catalizadas enzimáticamente que incluyen la formación de pigmentos y compuestos aromáticos y saborizantes a partir de sustancias precursoras presentes en el fruto maduro. El beneficiado consta de diversas operaciones, incluyendo recepción, despezonado, enmaletado, matado de fruto, asoleado, sudado, depósito y empaque. Dependiendo del grado de incorporación al proceso de maquinaria adecuadamente diseñada y calibrada, el beneficiado se clasifica como tradicional, semi apariencia (gruesa, delgada) y aroma (dulce, característico) (Reyes-López et al., 2008). Existen pocos trabajos que muestren los perfiles químicos de extractos de vainilla debido a que en la mayoría de las investigaciones solo se determina a los grupos funcionales y no se caracteriza a los componentes químicos específicos de loa extractos. Pase a esto, en las vainas de vainilla se ha repostado ampliamente a las vainas de la vainillina como el componente químico volátil más importante en la caracterización química, ya que la mayor concentración de esta se ha relacionado con una mejor calidad de sabor y aroma en las vainas curadas y sus extractos. Sin embargo, existen algunos perfiles químicos que reflejan una gran cantidad de componentes, semivolátiles o no volátiles que otorgan un papel significativo a estos compuestos al impartir a la vainilla o a sus extractos aroma y sabor característico (Sun et al., 2001; John y Jamin, 2004; Rocha et al., 2007; Sinha et al., 2008). Las esencias o extractos de vainilla natural se elaboran picando finamente vainas de vainilla y colocándolos en maceración o infusión en mezclas de alcohol y agua. El proceso generalmente se mantiene a temperatura ambiente (de preferencia frio) para reducir las pérdidas del sabor, a un que algunos fabricantes sugieren que la aplicación de calor favorece la extracción (Gatfield et al., 2007). La vainilla como saborizante se puede dividir en tres categorías: Categoría 1: Corresponde a un extracto de vainilla natural, los alimentos a los que se les adiciona este tipo de extractos son etiquetados como alimentos de vainilla. Categoría 2: Es un producto de vainillavainillina considerando un saborizante naturalartificial, en el que el componente natural aporta el sabor a vainilla característico. Los productos alimenticios a los que se les adiciona este extracto deben ser etiquetados como alimentos saborizados con vainilla. Categoría 3: Corresponde a saborizantes de vainillina artificial y los productos que los contiene deben ser etiquetados como alimentos de vainilla saborizados artificialmente (Sachan, 2005).

El objetivo del proyecto fue Evaluar el proceso tradicional de elaboración de extracto de Vainilla (Vanilla planifolia A.) para detectar posibles áreas de oportunidad y su correcto uso como generador de ingresos económicos alternativos al productor.

\section{MATERIALES Y MÉTODOS}

Las muestras de Vainilla se obtuvieron estación de campo Sierra de Otontepec que se ubica en el municipio de Chontla (21 ${ }^{\circ} 14^{\prime} 36.6^{\prime}$ ' y $21^{\circ} \mathrm{N}$, y $97^{\circ} 56^{\prime} 35.7^{\prime}$ ' O), al norte de Veracruz, sobre la carretera rural Las Cruces-San Juan Otontepec, en la vertiente occidental del Área Natural Protegida Sierra de Otontepec (Rodríguez-Luna et al., 2011).

\section{Revista Científica Biológico Agropecuaria Tuxpan 6 (2)} ISSN: 2007-6940 


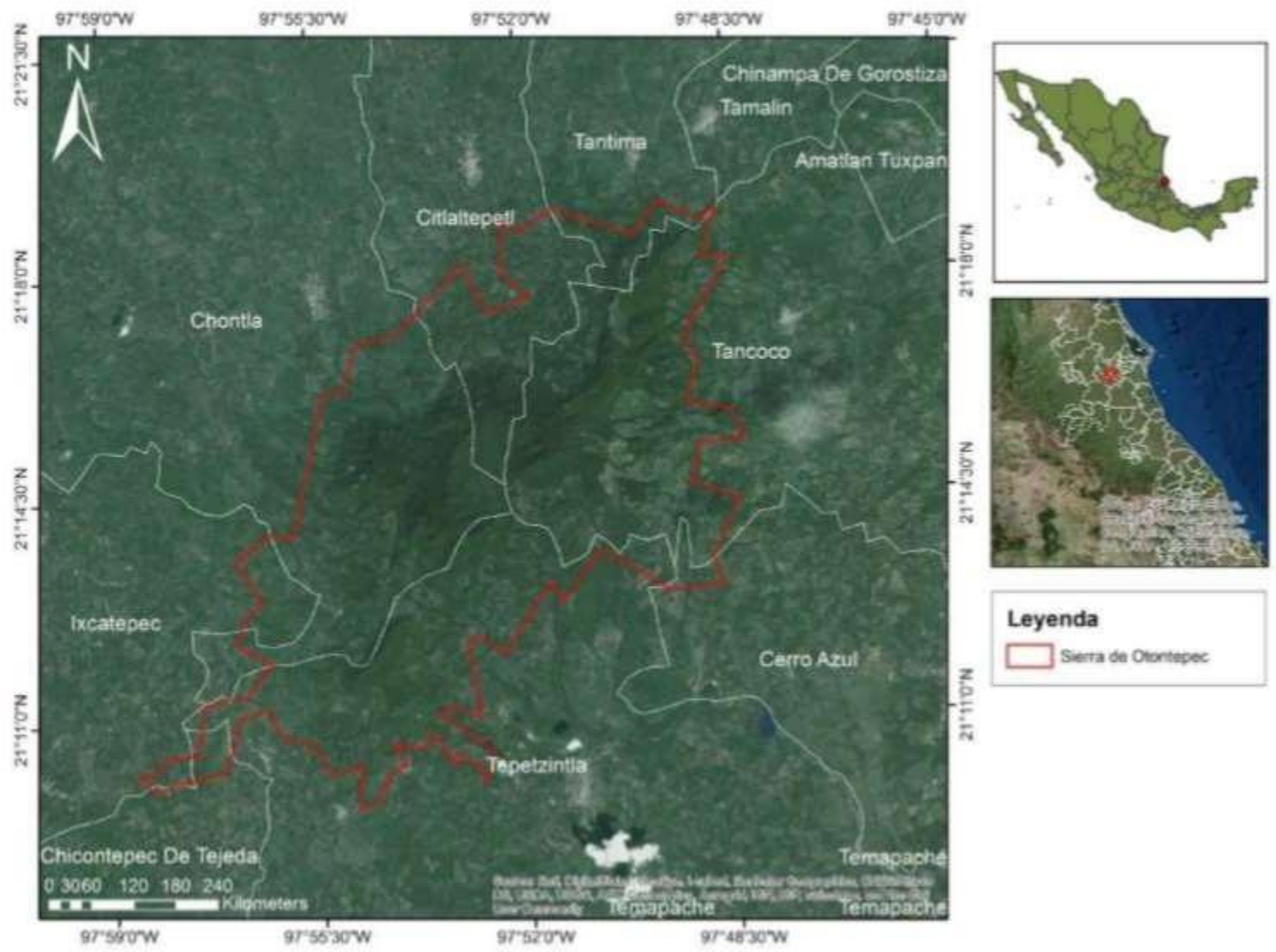

Figura 1. Área de estudio, estación de campo Sierra de Otontepec, en el municipio de Chontla, Veracruz.

Las muestras fueron llevadas al laboratorio de investigaciones de la Facultad de Ciencias Químicas, región Poza Rica Tuxpan de la Universidad Veracruzana. A las muestras se le cortaron las puntas de la vaina de cada extremo, posteriormente la vaina fue abierta y cortada a la mitad, fueron pesadas en una báscula y depositadas en un envase de vidrio $750 \mathrm{ml}$. Se llenó el frasco con refino artesanal asegurándo de que las vainas quedaran cubiertas por el líquido, se tapó y se agitó guardándolo en un sitio protegido de la luz. Se dejó reposar un mínimo de 3 meses. Se analizó el contenido de vainillina a los 3 meses de reposo, determinándose el contenido de vainillina del extracto por el método de espectrofotográfico NF (Method National Formulary) de la NOMFF-074-1996.

\section{RESULTADOS}

La primer variable de respuesta evaluada fue la identificación de vainillina presente en los extractos de refino artesanal mediante cromatografía líquida de alta eficacia (HPLC) bajo las siguientes condiciones: 
horno a temperatura de $40^{\circ} \mathrm{C}$, bomba cuaternaria con un flujo de $2.5 \mu \mathrm{L} / \mathrm{min}$ de una mezcla de Metanol/vainillina/100PPM/25Mg, con una frecuencia de obtención de datos de $5 \mathrm{~Hz}$, inyector automático estándar de $10 \mathrm{uL}$, obteniendo los siguientes resultados la muestra 6 presento el mayor porcentaje con 8,447 ppm a diferencia de la muestra 5 que presentó el menor porcentaje con 348 PPM, estas diferencias se pueden deber a las tonalidades y el tiempo de fermentación de cada extracto lo que coincide con lo propuesto por Waliszewski et al. (2007) al usar el método ( HPLC) en la concentración de vainillina del mismo modo coincidiendo en lo establecido en la NOM- FF- 074-1996 la cual establece en el apartado 5.2 que debe tener un $2,49 \%$ de vainillina en adelante así mismo menciona las especificaciones mínimas de calidad que debe cumplir la vainilla (Vanilla planifolia Andrews), en sus diferentes variedades, perteneciente a la familia de las orquidáceas, para ser comercializada y consumida en estado seco en territorio nacional, después de su beneficiado (deshidratación, sudado y secado) y envasado. la segunda variable de respuesta la cual fue un estudio del contenido de vainillina del extracto por el método espectrofotométrico NF (Method National Formulary), en el cual se llevó a cabo de la siguiente manera, se determinó la absorbencia de las soluciones alcalinas a 348 $\mathrm{nm}$, utilizando las soluciones testigo como blancos de referencia, en este método solo se utilizaron 5 muestras ya que es la cantidad mínima de calibración en la norma establecida, se preparó la curva estándar en la cual se trazó la curva patrón, graficando concentración vainillina contra absorbencia obteniendo los siguientes resultados: La muestra 3 presentó el mayor porcentaje de $19.756 \mathrm{C} / \mathrm{A}$, la muestra 1 presentó el menor porcentaje $3.170 \mathrm{C} / \mathrm{A}$ esto se puede deber a las diferentes concentraciones de vainillina sin embargo las concentraciones concuerdan con lo establecido en la NOM- FF0741996 la cual menciona en el apartado 5.2.1 que debe presentar un 2,5 de C/A en adelante de vainillina.

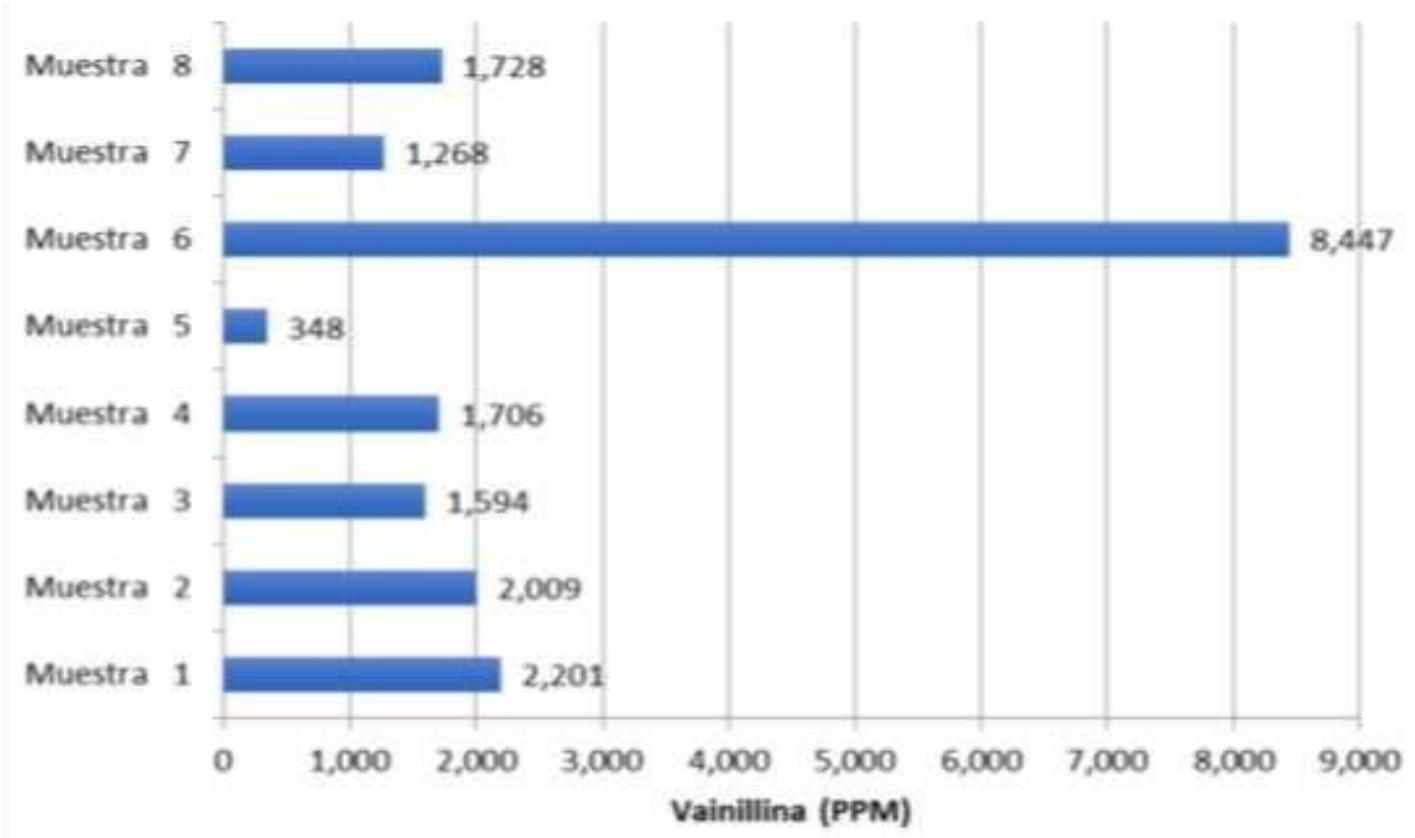

Figura 2. Concentración de vainilla en extracto artesanal.

Revista Científica Biológico Agropecuaria Tuxpan 6 (2) 
Con respecto al refino, los resultados muestran que el utilizado en la zona no es el ideal para elaborar extracto debido a que no contiene el \% de alcohol que la NMX-FF-074-SCFL-2009 establece. Misma que nos dice que la concentración de alcohol no debe ser menor del $35 \%$ y el refino cuenta con $14 \%$.

\section{CONCLUSIÓNES}

En base al análisis de los resultados se determinó que el refino utilizado en la zona no cuenta con el porcentaje de alcohol establecido en la NMXFF074-SCFL-2009.

El análisis de los resultados nos muestra que para el caso de los extractos hechos con refino artesanal se deben analizar por el Método de Cromatografía de alta resolución (HPLC), Método de Espectro fotográfico para obtener una concentración óptima de la calidad de vainillina. Se deberá buscar alternativas de asesoría para que los productores de vainilla tengan un uso adecuado de la elaboración del extracto artesanal.

\section{LITERATURA CITADA}

Curti D. E. 1995b. Cultivo y beneficio de la vainilla en México. Organización Nacional de Vainilleros Indígenas. Papantla. Ver. P. 96.

Curti, G. 1995b. Evaluación del cultivo y producción de vainilla en la Zona de Papantla Veracruz. Tesis de Doctorado. Instituto de Ecología, A.C. Xalapa, Veracruz. 79.p.

Gatfield, I. 2007. Ingredientes al día; La biotecnología de los aromas. Industria Alimentaria 20-58.www.industriaalimentaria.

Gatfield, 1., Hilmer.J, Weber, B., Hammerschmidt, F., Recib, I., Poutot, G., Bertrand, H., Meier D.
2007. Chemical and Biochemical changes ocurring during the traditional Madagascar vainilla curing process. Perfumer \& Flavor. $32: 1-8$

John, T.V. y Jamin, E. 2004. Investigación química y autenticidad de vainas de vainilla India. Revista de Quimica Agrícola y Alimentaria. 52:7644-7650.

Ranadive A. (1992). Vanillin and related flavor compound in vanilla in vanilla extracts made from beans of various global origins. Journal of Agricultural and Food Chemistry 40:19221924. https://doi.org/10.1021/jf00022a039

Reyes-López D., Rodríguez Morales, B., Kelso Bucio H., Huerta Lara M., Ibáñez Martínez A. 2008. Beneficiado tradicional de vainilla. México. Benemérita Universidad Autónoma de Puebla. Puebla, México.

Rodríguez-Luna, E., A. Gómez-Pompa., J.C. LópezAcosta., N. Velázquez., Y. Aguilar y M. Vázquez. 2011. Atlas de los espacios naturales protegidos de Veracruz. Gobierno del Estado de Veracruz, Secretaría de Educación del Estado de Veracruz, UV, Centro de Investigaciones Tropicales. 350 pp. Sachan, D. 2005. Vainilla y su Potencial en la India. Brief on New Publication.17:14. 
SAGARPA (Servicio de Información Agroalimentaria y Pesquera).2010.Mejora INIFAP tecnología para producción. Boletin.o67. Septiembre 04.

Soto, A. M. A. 2006. La vainilla: retos y perspectivas de su cultivo (CONABIO), Biodiviersitas 66:1-9.

Soto, A. M. A. 2009. Recopilación y análisis de la información existente sobre las respuestas mexicanas del género Vanilla. Reporte a la Comisión Nacional para el Conocimiento y
Uso de la Biodiversidad. (CONABIO), México, D.F.

Sun, R., Sacalis, J., Chin, C. y Still C. 2001. Bioactive Aromatic Compounds from Leaves and Stems of Vanilla fragrans. Journal of Agricultural and Food Chemistry 49:51615164. https://doi.org/10.1021/jf010425k

Waliszewski, K.N., Pardío, V.T., Ovando, S.L. 2007. A simple and rapid HPLC technique for vanillin determination in alcohol extract. Food Chemistry 101:1059-1062. https://doi.org/10.1016/j.foodchem.2006.03.0 04

Copyright (c) 2018 C. D. Solares del A, Pablo Elorza Martinez, B. A. Rodriguez, R. D. López C. y E. Aguirre López

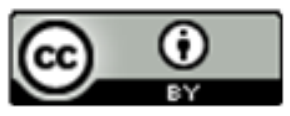

Este tex to está protegido por una licencia Creative Commons 4.0

Usted es libre para Compartir —copiar y redistribuir el $\mathrm{m}$ aterial en cualquier medio o format o- $\mathrm{y}$ Adaptar el documento - remezclar, transformar y crear a partir del material- para cualquier propósito, incluso para fines comerciales, siempre que cumpla la condición de:

Atribución: Usted debe dar crédito a la obra original de manera adecuada, proporcionar un enlace a la licencia, e in dicar si se han realizado cambios. Puede hacerlo en cualquier forma razonable, pero no de forma tal que sugiera que tiene el apoyo del licenciante o 10 recibe por el uso que hace de la obra.

Resumenclelicencia - Textocompletocielalicencia

Revista Científica Biológico Agropecuaria Tuxpan 6 (2) 